\title{
Influence of the electric induction drag on the orbit of a charged satellite moving in the ionosphere (solution by the method of the average value)
}

\author{
Lin-Sen Li ${ }^{1}$
}

Received: 23 April 2015 / Accepted: 17 November 2015 / Published online: 1 December 2015

(C) The Author(s) 2015. This article is published with open access at Springerlink.com

\begin{abstract}
The secular effects of the electric induction drag on the orbit of a charged satellite moving in the ionosphere are examined by the method of average values. The first solutions are obtained under the assumption of non-rotation of the Earth; the second solutions are obtained assuming rotation of the Earth. In the first case the semi-major axis exhibits secular variation, but the other orbital elements exhibit no secular variation. In the second case both semi-major axis and eccentricity exhibit secular variation, but the other orbital elements exhibit no secular variation. It can be shown that the semi-major axis is contracted due to the action of the electric induction drag if the satellite has enough charge in the ionosphere. The eccentricity is decreased gradually with time, but its variation is very small for the case of a rotating Earth. An example is presented in which the secular effects of the electric induction drag on the orbits of a charged satellite are calculated. The numerical results are given in Table 1 and a discussion of them is presented in Table 2.
\end{abstract}

Keywords Electric induction drag · Ionosphere - Charged satellite - Orbital influence

\section{Introduction}

As an artificial satellite pass through the ionosphere where there exists a great number of electrons and ions, these particles ions and electronics collide with the satellite to produce a drag. This drag is termed Coulomb drag. Chapra (1961) gave a formula for Coulomb drag. The author Li (2011a)

$凶$ L.-S. Li

dbsd-1ls@163.com

1 School of Physics, Northeast Normal University, Changchun, 130024, China studied the effect of Coulomb drag on the orbital elements of an artificial satellite moving in the ionosphere. If the satellite is electrically charged or the satellite is a metallic conductor while in the ionosphere, the mutual action of the charged satellite with the electric field produces an electric induction drag. Waytt (1960) obtained the formula for the electric induction drag, but that paper did not determine of it on the orbit of a charged satellite. The present paper studies the secular effect of the electric induction drag on the orbit of a charged satellite by using the method of average value.

\section{The electric induction drag and the components of the perturbation acceleration of a charged satellite on the elliptic orbit}

Waytt (1960) gave the formula of the electric induction drag force firstly

$$
F=-\int \nabla \phi\left(r^{\prime}\right) \rho_{e} d r^{\prime}
$$

He derived the solution of the potential function $\phi\left(r^{\prime}\right)$ from Boltzmann transport equation, and then he obtain the formula of the electric induction drag force $F$ on a charged satellite moving in a magnetic field free ionosphere with altitude $500 \mathrm{~km}$

$F=-5 Q^{2} B_{S} / 48 \pi a_{S}^{2} R_{S}^{2}$

Where $Q$, and $R_{S}$ denote respectively the charge and radius of the satellite and

$$
\begin{aligned}
& B_{S}=\sqrt{\pi A_{S-}} V / \lambda_{D-}^{2}, \quad A_{S_{-}}=m_{e} / 2 \theta_{-}, \\
& \theta_{-}=K T_{e}
\end{aligned}
$$




$$
\begin{aligned}
& a_{S}^{2}=1 / \lambda_{D-}^{2}+2 / \lambda_{D_{+}}^{2}, \quad \lambda_{D-}^{2}=K T_{e} / 4 \pi N_{e} e^{\prime 2}, \\
& \lambda_{D+}^{2}=K T_{i} / 4 \pi N_{e} e_{i}^{\prime 2} .
\end{aligned}
$$

We let $V=v-v_{E}$ (the velocity of the satellite relative to the ionosphere), where $v$ denotes the velocity of the satellite relative to the Earth's center. $n_{E}$ is the linear velocity of the rotation of the ionosphere. Here $m_{e}, T_{e}$ denote the mass and temperature of an electron respectively. $m_{i}, T_{i}$ denote mass and temperature of an ion respectively. $e^{\prime}$ denotes the charge of an electron. $N_{e}$ denotes the number density of the electron, and. $K$ is Boltzmann's constant.

Substituting expressions (2a), (2b) into the formulae (1), we obtain formula for the drag $F$ and acceleration $a_{c}$

$F=-\frac{5}{48} c\left(\frac{Q}{R_{S}}\right)^{2}\left(v-v_{E}\right)$

$a_{c}=-\frac{5}{48} c\left(\frac{Q^{2}}{m_{S} R_{S}^{2}}\right)\left(v-v_{E}\right)$

Where $m_{S}$ is the mass of the satellite and $c$ is the constant

$c=\sqrt{\frac{m_{e}}{2 \pi K T_{e}}} /\left(1+2 \frac{T_{e}}{T_{i}}\right)$.

Next, we deduce three perturbation components of the electric induction drag on the orbit of the charged Satellite.

The direction of the electric induction drag is in opposition to the direction of the orbital motion of the satellite. The drag acceleration is tangential. Writing the three perturbation components $U, N, W$ for the tangential, normal and the perpendicular direction to the motion of the charged satellite, we therefore have

$$
\left\{\begin{array}{l}
U=-\frac{5}{48} c Q^{2}\left(v-v_{E}\right) / m_{S} R_{S}^{2}, \\
N=0, \\
W=0 .
\end{array}\right.
$$

From Kepler's third law $n^{2} a^{3}=G M_{E}$, and $r=a(1-$ $\left.e^{2}\right) /(1+e \cos f)$, we have

$v=n a^{3 / 2} \sqrt{\left(1+2 e \cos f+e^{2}\right) / a\left(1-e^{2}\right)}$.

The relation between the rotational velocity of the atmosphere $\omega_{A}$ at $r$ and the rotational velocity of the Earth $\Omega_{E}$, are (Lamb 1932; Li 1991)

$\omega_{A}=\left(R_{E} / r\right)^{3} \Omega_{E}$.

Where $R_{E}, M_{E}$ denote the radius and mass of the Earth respectively. Hence, when we consider the effect of the rotation of the Earth, the linear velocity of the ionosphere at distance $r$ is

$$
\begin{aligned}
v_{E} & =r \omega_{A} \cos i=R_{E}^{3} \Omega_{E} \cos i / r^{2} \\
& =(1+e \cos f)^{2} R_{E}^{3} \Omega_{E} \cos i / a^{2}\left(1-e^{2}\right)^{2},
\end{aligned}
$$

where $i$ is the orbital inclination of the satellite. If we do not consider the effect of the rotation of the Earth,

$v_{E}=0$.

Substituting $v$ and $v_{E}$ into the expressions (6), we obtain

$$
\left\{\begin{aligned}
U= & -\frac{5 c Q^{2}}{48 m_{S} R_{S}^{2}}\left[n a^{3 / 2} \sqrt{\frac{1+e^{2}+2 e \cos f}{a\left(1-e^{2}\right)}}\right. \\
& \left.-\frac{(1+e \cos f)^{2} R_{E}^{3} \Omega_{E} \cos i}{a^{2}\left(1-e^{2}\right)^{2}}\right], \\
N= & 0, \\
W= & 0 .
\end{aligned}\right.
$$

Substitution of $U, N, W$ into the formulae of three components, $S, T, W$ for the radial, transverse, and normal directions (Roy 1988), we obtain

$$
\begin{aligned}
S= & \frac{e \sin f}{\sqrt{1+e^{2}}+2 e \cos f} U-\frac{1+e \cos f}{\sqrt{1+e^{2}+2 e \cos f}} N \\
= & -\frac{5 c Q^{2}}{48 m_{S} R_{S}^{2}}\left[\frac{n a e \sin f}{\sqrt{1-e^{2}}}\right. \\
& \left.-\frac{(1+e \cos f)^{2} e \sin f R_{E}^{3} \Omega_{E} \cos i}{a^{2}\left(1-e^{2}\right)^{2} \sqrt{1+e^{2}+2 e \cos f}}\right] \\
= & S_{N R}+S_{R}, \\
T= & \frac{1+e \cos f}{\sqrt{1+e^{2} 2 e \cos f} U+\frac{e \sin f}{\sqrt{1+e^{2}+2 e \cos f}}} N \\
= & -\frac{5 c Q^{2}}{48 m_{S} R_{S}^{2}}\left[\frac{n a(1+e \cos f)}{\sqrt{1-e^{2}}}\right. \\
& \left.-\frac{(1+e \cos f)^{3} R_{E}^{3} \Omega_{E} \cos i}{a^{2}\left(1-e^{2}\right)^{2} \sqrt{1+e^{2}+2 e \cos f}}\right] \\
= & T_{N R}+T_{R}, \\
W= & W_{N R}+W_{R}=0 .
\end{aligned}
$$

Here the subscripts $N R$ and $R$ denote non-rotation and rotation of the Earth respectively.

Here the symbols $a$ and $e$ denote the semi-major axis and eccentricity respectively, $n$ is the mean motion, and $f$ is the true anomaly. The second terms in the right hand show the effect of the rotation of the Earth. 


\section{Solution for the case of non-rotation of the Earth and orbital inclination of the satellite}

For the case of non-rotation of the Earth we designate certain terms in the expressions (10)

$$
\left\{\begin{array}{l}
S_{N R}=-\frac{5 c Q^{2}}{48 m_{S} R_{S}^{2}}\left(\frac{n a e \sin f}{\sqrt{1-e^{2}}}\right), \\
T_{N R}=-\frac{5 c Q^{2}}{48 m_{S} R_{S}^{2}}\left(\frac{n a(1+e \cos f)}{\sqrt{1-e^{2}}}\right) \\
W_{N R}=0 .
\end{array}\right.
$$

Substitution of the formulae (11) into the Gaussian perturbation Gaussian equations (Roy 1988)

$$
\left\{\begin{aligned}
\frac{d a}{d t} & =\frac{2}{n \sqrt{1-e^{2}}}[S e \sin f+T(1+e \cos f)], \\
\frac{d e}{d t} & =\frac{\sqrt{1-e^{2}}}{n a}[S \sin f+T(\cos E+\cos f)], \\
\frac{d i}{d t} & =\frac{r \cos u}{n a^{2} \sqrt{1-e^{2}}} W, \\
\frac{d \Omega}{d t} & =\frac{r \sin u}{n a^{2} \sqrt{1-e^{2}} \sin i} W, \\
u & =\varpi+f . \\
\frac{d \omega}{d t} & =\frac{\sqrt{1-e^{2}}}{n a e}\left[-S \cos f+T\left(1+\frac{r}{p}\right) \sin f\right], \\
\frac{d \varpi}{d t} & =\frac{d \omega}{d t}+\frac{d \Omega}{d t}, \\
\frac{d \varepsilon_{0}}{d t} & =-\frac{2 r}{n a^{2}} S+\left(\frac{e^{2}}{1+\sqrt{1-e^{2}}}\right) \frac{d \varpi}{d t} .
\end{aligned}\right.
$$

From these, we obtain

$$
\left\{\begin{array}{l}
\frac{d a}{d t}=-\frac{5 c Q^{2}}{24 m_{S} R_{S}^{2}} \frac{a\left(1+e^{2}+2 e \cos f\right)}{1-e^{2}}, \\
\frac{d e}{d t}=-\frac{5 c Q^{2}}{24 m_{S} R_{S}^{2}}(e+\cos f), \\
\frac{d \varpi}{d t}=\frac{d \omega}{d t}=-\frac{5 c Q^{2}}{24 m_{S} R_{S}^{2}}\left(\frac{\sin f}{e}\right), \\
\frac{d \varepsilon_{0}}{d t}=\frac{5 c Q^{2}}{24 m_{S} R_{S}^{2}}\left(\frac{r}{a \sqrt{1-e^{2}}}-\frac{1}{1+\sqrt{1-e^{2}}}\right) e \sin f .
\end{array}\right.
$$

In this paper we use the method of average for $\sin i f$ and cos if as listed in the table given by Liu (1992)

$$
\left\{\begin{aligned}
\overline{\sin f} & =\frac{1}{2 \pi} \int_{0}^{2 \pi} \sin f d M \\
& =\frac{1}{2 \pi} \int_{0}^{2 \pi} \sin f\left(\frac{r}{a}\right) d E \\
& =\frac{1}{2 \pi} \int_{0}^{2 \pi} \sqrt{1-e^{2}} \sin E d E \\
& =0 \\
\overline{\cos f} & =\frac{1}{2 \pi} \int_{0}^{2 \pi} \cos f d M \\
& =\frac{1}{2 \pi} \int_{0}^{2 \pi} \cos f\left(\frac{r}{a}\right) d E \\
& =\frac{1}{2 \pi} \int_{0}^{2 \pi}(\cos E-e) d E \\
& =-e .
\end{aligned}\right.
$$

Substituting the average values for $\overline{\sin f}$ and $\overline{\cos f}$ into the average from equations (13), we get

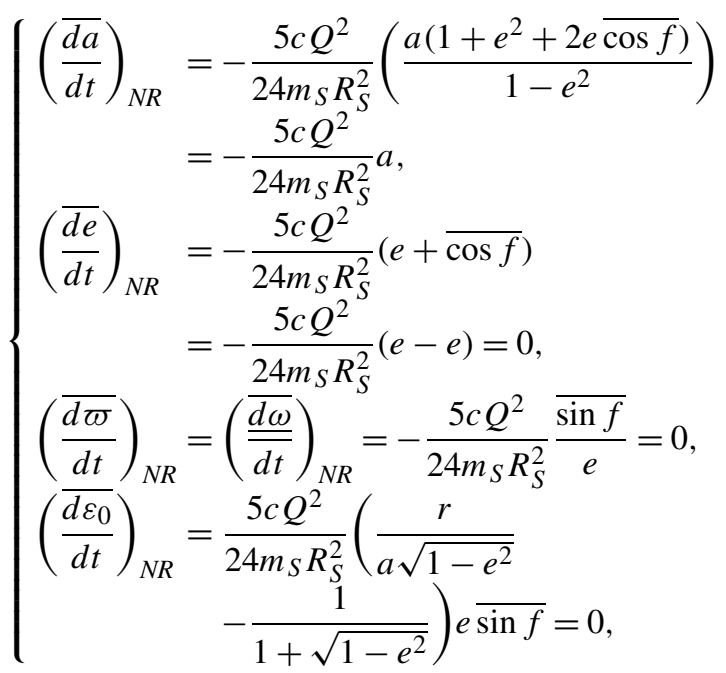

According to Kepler's third law, $n^{2} a^{3}=G M_{E}$ and we obtain

$$
\left(\frac{d T_{S}}{d t}\right)_{N R}=\frac{3}{2} \frac{T_{S}}{a}\left(\frac{d a}{d t}\right)_{N R}=-\frac{15}{48 m_{S}} \frac{c Q^{2}}{R_{S}^{2}} T_{S} .
$$

Where $T_{S}$ denotes the orbital period.

\section{Solution for the case of rotation of the Earth and inclination of the orbit of the satellite}

First, we expand the expression

$$
\left(1+e^{2}+2 e \cos f\right)^{-1 / 2}
$$




$$
\begin{aligned}
& =\left[\left(1+e^{2}\right)\left(1+\frac{2 e \cos f}{1+e^{2}}\right)\right]^{-1 / 2} \\
& =\frac{1}{\sqrt{1+e^{2}}}(A+B \cos f+C \cos 2 f),
\end{aligned}
$$

where

$A=1+\frac{3}{4}\left(\frac{e}{1+e^{2}}\right)^{2}, \quad B=-e /\left(1+e^{2}\right)$,

$C=\frac{3}{4}\left(\frac{e}{1+e^{2}}\right)^{2}$.

Substituting the above expressions into the second term in the right hand of Eq. (10) and using $1+e \cos f / a\left(1-e^{2}\right)=$ $1 / r$, we designate certain terms as before

$$
\left\{\begin{aligned}
S_{R}= & \left(\frac{5 c Q^{2}}{48 m_{S} R_{S}^{2}}\right) \frac{e \sin f R_{E}^{3} \Omega_{E} \cos i}{r^{2} \sqrt{1+e^{2}}} \\
& \times(A+B \cos f+C \cos 2 f), \\
T_{R}= & \left(\frac{5 c Q^{2}}{48 m_{S} R_{S}^{2}}\right) \frac{R^{3} p \Omega_{E} \cos i}{r^{3} \sqrt{1+e^{2}}} \\
& \times(A+B \cos f+C \cos 2 f), \\
W_{R}= & 0,
\end{aligned}\right.
$$

where $p=a\left(1-e^{2}\right)$.

Substituting $S, T, W$ from the formulae (17) into the Gaussian equation (12), we get

$$
\begin{aligned}
\frac{d a}{d t}= & \frac{5 c Q^{2}}{48 m_{S} R_{S}^{2}} \frac{2 R_{E}^{3} \Omega_{E} \cos i}{n \sqrt{1-e^{2}} \sqrt{1+e^{2}}}\left(\frac{e^{2} \sin ^{2} f}{r^{2}}-\frac{p^{2}}{r^{4}}\right) \\
& \times(A+B \cos f+C \cos 2 f) .
\end{aligned}
$$

Using $\sin ^{2} f=\frac{1}{2}\left(1-\cos ^{2} f\right)$, we obtain

$$
\begin{aligned}
\frac{\overline{d a}}{d t}= & \frac{5 c Q^{2}}{48 m_{S} R_{S}^{2}} \frac{2 R_{E}^{3} \Omega_{E} \cos i}{n \sqrt{1+e^{2}} \sqrt{1-e^{2}}} \\
& \times\left\{\frac{1}{2} e^{2} \frac{A}{a^{2}} \overline{\left(\frac{a}{r}\right)^{2}}+\frac{1}{2} e^{2} \frac{B}{a^{2}} \overline{\left(\frac{a}{r}\right)^{2} \cos f}\right. \\
& +\frac{1}{2} C\left(\frac{e}{a}\right)^{2} \overline{\left(\frac{a}{r}\right)^{2} \cos 2 f} \\
& -\frac{1}{2} A\left(\frac{e}{a}\right)^{2}\left(\frac{a}{r}\right)^{2} \cos 2 f \\
& -\frac{1}{2} B\left(\frac{e}{a}\right)^{2} \overline{\left(\frac{a}{r}\right)^{2} \cos f \cos 2 f}
\end{aligned}
$$

$$
\begin{aligned}
& -\frac{1}{2} C\left(\frac{e}{a}\right)^{2} \overline{\left(\frac{a}{r}\right)^{2} \cos ^{2} 2 f}+A \frac{p^{2}}{a^{4}} \overline{\left(\frac{a}{r}\right)^{4}} \\
& \left.+B \frac{p^{2}}{a^{4}} \overline{\left(\frac{a}{r}\right)^{4} \cos f}+C \frac{p^{2}}{a^{4}}\left(\frac{a}{r}\right)^{4} \cos 2 f\right\} .
\end{aligned}
$$

$\overline{\left(\frac{a}{r}\right)^{2}}=\left(1-e^{2}\right)^{-1 / 2}, \quad \overline{\left(\frac{a}{r}\right)^{2} \cos f}=0$,

$\overline{\left(\frac{a}{r}\right)^{2} \cos 2 f}=0, \quad \overline{\left(\frac{a}{r}\right)^{2} \cos f \cos 2 f}=0$,

$\overline{\left(\frac{a}{r}\right)^{2} \cos ^{2} 2 f}=\left(1-e^{2}\right)^{1 / 2}$,

$\overline{\left(\frac{a}{r}\right)^{4}}=\left(1+\frac{1}{2} e^{2}\right)\left(1-e^{2}\right)^{-5 / 2}$,

$\overline{\left(\frac{a}{r}\right)^{4} \cos f}=e\left(1-e^{2}\right)^{-5 / 2}$,

$\overline{\left(\frac{a}{r}\right)^{4} \cos 2 f}=\frac{1}{4} e^{2}\left(1-e^{2}\right)^{-5 / 2}, \quad \frac{p^{2}}{a^{4}}=\frac{\left(1-e^{2}\right)}{a^{2}}$.

Substituting the results of the average values into Eq. (18), we find that

$$
\begin{aligned}
\frac{\overline{d a}}{d t}= & \frac{5 c Q^{2}}{48 m_{S} R_{S}^{2}} \frac{2\left(1-e^{2}\right)^{-1 / 2}}{\sqrt{1+e^{2}} \sqrt{1-e^{2}} n a^{2}}\left\{\frac{1}{2} e A-\frac{1}{4} e^{2} C\right. \\
& \left.+\left(1+\frac{1}{2} e^{2}\right) A+B e+\frac{1}{4} C e\right\} R^{3} \Omega \cos i .
\end{aligned}
$$

Substituting the above values and the values of $A, B, C$ that we used in the expressions (16) into Eq. (18), and neglecting higher than $e^{3}$ terms, and using $n a^{2}=\sqrt{G M_{E} a}$, we get

$$
\left(\frac{d a}{d t}\right)_{R}=\frac{5 c Q^{2}}{48 m_{S} R_{S}^{2}} \frac{\left(1+\frac{3}{4} e^{2}\right) R_{E}^{3} \Omega_{E} \cos i}{\sqrt{G M_{E} a}\left(1-e^{2}\right)\left(1+e^{2}\right)^{1 / 2}} .
$$

Averaging over the Gaussian equation for variable rate of eccentricity, we have

$$
\begin{aligned}
\frac{d e}{d t}=\frac{\sqrt{1-e^{2}}}{n a} & {[S \sin f+T(\cos E+\cos f)], } \\
\cos E+\cos f & =\frac{\cos f+e}{1+e \cos f}+\cos f \\
& =\frac{r}{p}\left(\frac{3}{2} e+2 \cos f+\frac{1}{2} e \cos 2 f\right) .
\end{aligned}
$$

Substituting the above expression with $S$ and $T$ from the expressions (17) into the Gaussian equation (12) for $\frac{d e}{d t}$, this 
equation can be written

$$
\begin{aligned}
\frac{d e}{d t}= & +\left(\frac{5 c Q^{2}}{48 m_{S} R_{S}^{2}}\right) R_{E}^{3} \Omega_{E} \cos i \frac{\sqrt{1-e^{2}}}{n a \sqrt{1+e^{2}}} \\
& \times(A+B \cos f+C \cos 2 f) \\
& \times \frac{1}{r^{2}}\left(e \sin ^{2} f+\frac{3}{2} e+2 \cos f+\frac{1}{2} e \cos 2 f\right) .
\end{aligned}
$$

Using $\sin ^{2} f=\frac{1}{2}(1-\cos 2 f)$, this becomes

$$
\begin{aligned}
\frac{d e}{d t}= & +\frac{5 c Q^{2}}{48 m_{S} R_{S}^{2}} R_{E}^{3} \Omega_{E} \cos i \frac{\sqrt{1-e^{2}}}{n a \sqrt{\left(1+e^{2}\right)} r^{2}} \\
& \times(A+B \cos f+C \cos 2 f)(2 e+2 \cos f) .
\end{aligned}
$$

Consequently

$$
\begin{aligned}
& \frac{\overline{d e}}{d t}=+\frac{5 c Q^{2}}{48 m_{S} R_{S}^{2}} R_{E}^{3} \Omega_{E} \cos i \frac{\sqrt{1-e^{2}}}{\sqrt{1+e^{2}}} \\
& \times \frac{2}{n a^{3}}\left[e A \overline{\left(\frac{a}{r}\right)^{2}}+e B \overline{\left(\frac{a}{r}\right)^{2} \frac{\cos f}{2}}\right. \\
& +e C \overline{\left(\frac{a}{r}\right)^{2} \cos 2 f}+\overline{A\left(\frac{a}{r}\right)^{2} \cos f} \\
& \left.+B \overline{\left(\frac{a}{r}\right)^{2} \cos 2 f}+C \overline{\left(\frac{a}{r}\right)^{2} \cos f \cos 2 f}\right] \text {. }
\end{aligned}
$$

Since

$$
\begin{aligned}
& \overline{\left(\frac{a}{r}\right)^{2}}=\left(1-e^{2}\right)^{-1 / 2}, \quad \overline{\left(\frac{a}{r}\right)^{2} \cos f}=0, \\
& \overline{\left(\frac{a}{r}\right)^{2} \cos 2 f}=0, \quad \overline{\left(\frac{a}{r}\right)^{2} \cos f \cos 2 f}=0, \\
& \overline{\cos ^{2} f}=\frac{1}{2} \overline{\left(\frac{a}{r}\right)^{2}}(1+\overline{\cos 2 f})=\frac{1}{2}\left(1-e^{2}\right)^{-1 / 2} .
\end{aligned}
$$

This expression becomes

$$
\begin{aligned}
\therefore \frac{\overline{d e}}{d t}= & +\left(\frac{5 c Q^{2}}{48 m_{S} R_{S}^{2}}\right) R_{E}^{3} \Omega_{E} \cos i \frac{\sqrt{1-e^{2}}}{\sqrt{1+e^{2}}} \frac{1}{n a^{3}} \\
& \times\left[A e\left(1-e^{2}\right)^{-1 / 2}+\frac{1}{2} B\left(1-e^{2}\right)^{-1 / 2}\right] .
\end{aligned}
$$

Substituting the values of $A$ and $B$ used in the expressions (16) and $n a^{3}=a^{3 / 2} \sqrt{G M_{E}}$ into the above equation and neglecting higher order terms than $e^{3}$, we obtain

$\therefore \overline{\left(\frac{d e}{d t}\right)_{R}}=+\frac{5 c Q^{2}}{48 m_{S} R_{S}^{2}} \frac{1}{\sqrt{G M_{E} a^{3}}} \frac{e}{\sqrt{1+e^{2}}} R_{E}^{3} \Omega_{E} \cos i$.
Substituting the components $S$ and $T$ from the expressions (17) into the Gaussian equation (12) for $\frac{d \omega}{d t}$, this equation becomes

$$
\begin{aligned}
\frac{d \omega}{d t}= & \frac{\sqrt{1-e^{2}}}{n a e}\left[-S \cos f+T\left(1+\frac{r}{p}\right) \sin f\right] \\
= & +\frac{5 c Q^{2}}{48 m_{S} R_{S}^{2}} R_{E}^{3} \Omega_{E} \cos i \frac{\sqrt{1-e^{2}}}{n a e \sqrt{1+e^{2}}} \\
& \times\left[\frac{e \sin f \cos f}{r^{2}}-\frac{P}{r^{3}}\left(1+\frac{r}{p}\right) \sin f\right] \\
& \times(A+B \cos f+C \cos 2 f) .
\end{aligned}
$$

Using $\sin f \cos f=\frac{1}{2} \sin 2 f$,

$$
\begin{aligned}
\frac{p}{r^{3}}\left(1+\frac{r}{p}\right) \sin f & =\frac{p}{r^{3}}\left(1+\frac{1}{1+e \cos f}\right) \sin f \\
& =\frac{p}{r^{3}}\left(\frac{2+e \cos f}{1+e \cos f}\right) \sin f \\
& =\frac{(2+e \cos f)}{r^{2}} \sin f \\
& =\frac{1}{r^{2}}\left(2 \sin f+\frac{1}{2} e \sin 2 f\right)
\end{aligned}
$$

It follows that

$$
\begin{aligned}
\frac{\overline{d \omega}}{d t}= & +\frac{5 c Q^{2}}{48 m_{S} R_{S}^{2}} \frac{R_{E}^{3} \Omega_{E} \cos i}{n a^{3} e} \frac{\sqrt{1-e^{2}}}{\sqrt{1+e^{2}}} \\
& \times\left[\frac{1}{2} e \overline{\left(\frac{r}{a}\right)^{2} \sin 2 f}-2 \frac{\left(\frac{a}{r}\right)^{2} \sin f}{1}\right. \\
& \left.-\frac{1}{2} e\left(\frac{a}{r}\right)^{2} \sin 2 f\right](A+B \cos f+C \cos 2 f) .
\end{aligned}
$$

Since $\overline{\left(\frac{a}{r}\right)^{2} \sin f}=0,\left(\frac{a}{r}\right)^{2} \sin 2 f=0$,

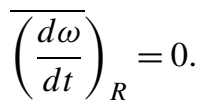

Similarly,

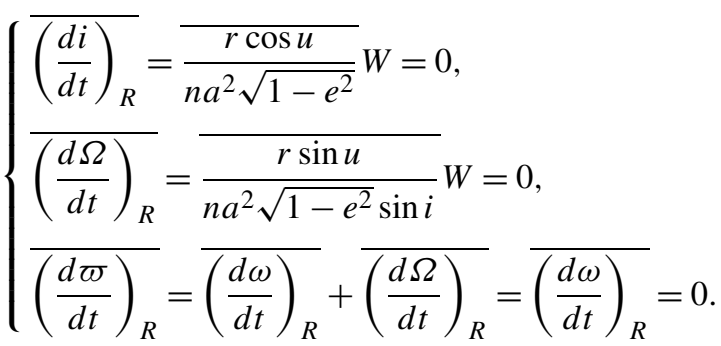


Substituting the component $S$ for the first expression of formula (17) into the following equation

$$
\begin{aligned}
\frac{d \varepsilon_{0}}{d t}= & -\frac{2}{n a^{2}} r S+\left(\frac{e^{2}}{1+\sqrt{1-e^{2}}}\right) \frac{d \varpi}{d t} \\
r S= & +\frac{2}{n a^{2}}\left(\frac{5 c Q^{2}}{48 m_{S} R_{S}^{2}}\right) \frac{e \sin f R_{E}^{3} \Omega_{E} \cos i}{r \sqrt{1+e^{2}}} \\
& \times(A+B \cos f+C \cos 2 f) .
\end{aligned}
$$

We obtain

$$
\begin{aligned}
& \therefore \frac{\overline{d \varepsilon_{0}}}{d t}=-\frac{5 c Q^{2}}{48 m_{S} R_{S}^{2}} R_{E}^{3} \Omega_{E} \cos i \frac{2}{n a^{3} \sqrt{1+e^{2}}} \\
& \times\left[A e \overline{\left(\frac{a}{r}\right) \sin f}+\overline{B e\left(\frac{a}{r^{2}}\right) \sin f \cos f}\right. \\
& \left.+C e \overline{\left(\frac{a}{r}\right) \sin f \cos 2 f}\right]+\frac{e^{2}}{1+\sqrt{1-e^{2}}} \frac{\overline{d \varpi}}{d t} \text {. }
\end{aligned}
$$

Since $\overline{\left(\frac{a}{r}\right) \sin f}=0, \overline{\left(\frac{a}{r}\right) \sin f \cos f}=\frac{1}{2} \overline{\left(\frac{a}{r}\right) \sin f \cos f}=$ $\frac{1}{2} \overline{\left(\frac{a}{r}\right) \sin 2 f}=0, \overline{\left(\frac{a}{r}\right) \sin f \cos 2 f}=0$, we arrive at

$$
\left(\overline{\frac{d \varpi}{d t}}\right)_{R}=0
$$

When we consider the rotation of the Earth and the inclination, we obtain

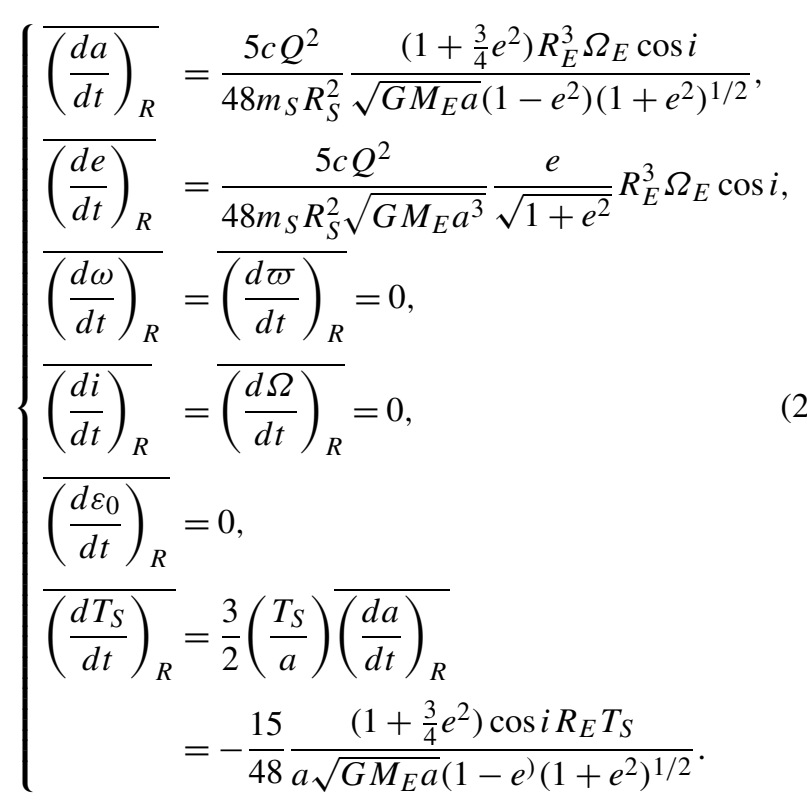

The total secular effects of the electric induction drag on the orbital elements of the charged satellite in the magnetic- free ionosphere are, therefore

$$
\left\{\begin{array}{l}
\overline{\dot{a}}=\overline{\dot{a}}_{N R}+\overline{\dot{a}}_{R}, \\
\overline{\dot{e}}=\overline{\dot{e}}_{N R}+\overline{\dot{e}}_{R}, \\
\overline{\dot{\omega}}=\overline{\dot{\omega}}_{N R}+\overline{\dot{\omega}}_{R}=0, \\
\overline{\dot{i}}=\overline{\dot{i}}_{N R}+\overline{\dot{i}}_{R}=0, \\
\overline{\dot{\Omega}}=\overline{\dot{\Omega}}_{N R}+\overline{\dot{\Omega}}_{R}=0, \\
\overline{\dot{\omega}}=\overline{\dot{\varpi}}_{N R}+\overline{\bar{\varpi}}_{R}=0, \\
\overline{\dot{\varepsilon}}_{0}={\overline{\left(\dot{\varepsilon}_{0}\right)}}_{N R}+{\overline{\left(\dot{\varepsilon}_{0}\right)}}_{R}+0, \\
{\overline{\dot{T}_{S}}}={\overline{\dot{T}_{S}}}_{N R}+{\overline{\dot{T}_{S}}}_{R} .
\end{array}\right.
$$

Waytt (1960) deems that the drag formula (1) is the approximate drag to consider for a satellite at altitude of $500 \mathrm{~km}$. As an example, for illustrating this work we consider a conductive satellite at an altitude of $500 \mathrm{~km}$ in the ionosphere. For this example $R_{S}=2 \mathrm{~m}, m_{S}=45 \mathrm{~kg}, T_{S}=102 \mathrm{~min}$, $a=7250 \mathrm{~km}$ and $e=0.025, i=75^{\circ}$. The satellite carries a charge $3 \mathrm{C}$ under the action of the electric induction as it moves through the ionosphere. Earth's mass $M_{E}=$ $5.976 \times 10^{27} \mathrm{~g}, R_{E}=6371 \mathrm{~km}, \Omega_{E}=7.2921 \times 10^{-5} \mathrm{rad} / \mathrm{s}$.

First, we must calculate the value of $c$ for the expression (4) at 500-km altitude in the ionosphere. According to data given by Allen (1973) the temperature of the electrons and ions at the height of $500 \mathrm{~km}$ in the ionosphere is respectively $T_{e}=1600 \mathrm{~K}, T_{i}=1600 \mathrm{~K}$, we are using $m_{e}=9.10956 \times$ $10^{-31}$ and $K=1.38062 \mathrm{Joule}^{-1} \mathrm{deg}^{-1}$. Substituting these data into expression (5), we obtain

$c=\sqrt{\frac{m_{e}}{2 \pi K T_{e}}} /\left(1+2 \frac{T_{e}}{T_{i}}\right)=5.26 \times 10^{-11}$.

Substituting into Eqs. (15) and (24), and then (15) and (24) into (25), we obtain the numerical results for effects of the orbital parameters of the charged satellite due to electric induction drag. This information is presented in Table 1.

In Case 1 the results are obtained from the formula (15), and in Case 2 the results are obtained from formula (24).

\section{Discussions and conclusions}

\subsection{Discussions}

(1) When we do not consider the rotation of the Earth, the results of Eqs. (15) give the variation of the orbital semimajor axis except all other orbital elements are without variation. When we consider the rotation of the Earth, the results of Eqs. (24) give the variation of the orbital semi-major axis and eccentricity: all other orbital elements are without variation. 
Table 1 Numerical results

\begin{tabular}{lllllllll}
\hline Effects & $\begin{array}{l}\dot{a} \\
(\mathrm{~m} / \mathrm{d})\end{array}$ & $\begin{array}{l}\dot{e} \\
(/ \mathrm{d})\end{array}$ & $\begin{array}{l}\dot{\omega} \\
(\mathrm{rad} / \mathrm{d})\end{array}$ & $\begin{array}{l}\dot{\Phi} \\
(\mathrm{rad} / \mathrm{d})\end{array}$ & $\begin{array}{l}\dot{1} \\
(\mathrm{rad} / \mathrm{d})\end{array}$ & $\begin{array}{l}\dot{\Omega} \\
(\mathrm{rad} / \mathrm{d})\end{array}$ & $\begin{array}{l}\dot{\varepsilon}_{0} \\
(\mathrm{rad} / \mathrm{d})\end{array}$ & $\begin{array}{l}\dot{T}_{S} \\
(\mathrm{~s} / \mathrm{d})\end{array}$ \\
\hline $\begin{array}{l}\text { Case 1 } \\
\text { Non-rotation }\end{array}$ & -8.36 & 0 & 0 & 0 & 0 & 0 & 0 & -0.01 \\
$\begin{array}{l}\text { Case 2 } \\
\text { Rotation }\end{array}$ & +0.13 & $2.15 \times 10^{-8}$ & 0 & 0 & 0 & 0 & 0 & $+1.65 \times 10^{-4}$ \\
$\begin{array}{l}\text { Sum } \\
\text { Total values }\end{array}$ & -8.23 & $+2.15 \times 10^{-8}$ & 0 & 0 & 0 & 0 & 0 & -0.0098 \\
\hline
\end{tabular}

(2) If we consider the case of rotation of the Earth and the inclination there exist variation of the eccentricity and of the semi-major axis even though their variation is very small, if the satellite moves along the equator of the Earth ( $\cos i=1)$, the effect is largest. If the satellite moves along a meridian circle $(\cos i=0)$, the perturbation effect is zero.

(3) In this paper we adopt the method of average value to study this topic because the factor $\left(1+e^{2}+\right.$ $2 e \cos f)^{-1 / 2}$ in the expressions (10) for the perturbation functions $S$ and $T$ becomes unwieldy, even when expanded. For this reason this paper uses the method of average value which is rather easy and simple.

(4) In this paper the orbital effects only consider electric field, the effects of the magnetic field are not considered. The charged or conductive satellite moves in the magnetic field-free ionosphere according to the formula of drag given by Waytt (1960)

(5) Comparison of the effect of electronic induction drag with that of neutral atmospheric drag at the same altitude.

If we consider neutral atmospheric drag, the variable rate of the semi-major axis may be calculated from the formula for the atmospheric drag (Zheng and Ni 1989)

$$
\begin{aligned}
\frac{d a}{d t}= & -\frac{2 \pi}{T_{S}} k_{S} a^{2} \rho_{0} \exp \left[\beta\left(r_{0}-a\right)\right] \\
& \times\left[I_{0}+2 e I_{1}+\frac{3}{4} e^{2}\left(I_{0}+I_{2}\right)+\cdots\right] \\
\frac{d e}{d t}= & -\frac{2 \pi}{T_{S}} k_{S} a \rho_{0} \exp \left[\beta\left(r_{0}-a\right)\right]\left[I_{0}+\frac{1}{2} e\left(I_{0}+I_{2}\right)\right. \\
& \left.+\frac{1}{4} e^{2}\left(-5 I_{1}+I_{3}\right) \ldots\right]
\end{aligned}
$$

and based on Kepler's third law

$$
\frac{d T_{S}}{d t}=\frac{3}{2} \frac{T_{S}}{a} \frac{d a}{d t}
$$

We consider the satellite at an altitude of $500 \mathrm{~km}$ and use data given by Allen (1973) $H=80, \beta=1 / 80$. $\log \rho_{0}=-15.2 \mathrm{~g} / \mathrm{cm}^{3}\left(\rho_{0}=3.60 \times 10^{-13} \mathrm{~kg} / \mathrm{m}^{3}\right), k_{S}=$
Table 2 Comparison of the electric induction drag with atmospheric drag and Coulomb drag

\begin{tabular}{llll}
\hline Effects & $\begin{array}{l}\text { Electric induction } \\
\text { drag }\end{array}$ & $\begin{array}{l}\text { Coulomb } \\
\text { drag }\end{array}$ & $\begin{array}{l}\text { Atmospheric } \\
\text { drag }\end{array}$ \\
\hline$\dot{a}(\mathrm{~m} / \mathrm{d})$ & -8.23 & -2.44 & -39.79 \\
$\dot{e}(/ \mathrm{d})$ & $0 \quad+2.15 \times 10^{-8}$ & $-2.11 \times 10^{-9}$ & $-5.42 \times 10^{-6}$ \\
$\dot{T}_{S}(\mathrm{~s} / \mathrm{d})$ & -0.0098 & -0.003 & -0.05 \\
\hline
\end{tabular}

$\lambda C_{D} S / m_{S}=0.5585 \mathrm{~m}^{2} / \mathrm{kg} \quad\left(\lambda=1, C_{D}=2.1, S=\right.$ $\left.\frac{1}{4} \pi R^{2}, m_{S}=45 \mathrm{~kg}\right), r_{0}=6371+500=6871 \mathrm{~km}$, $R_{E}=6371 \mathrm{~km}, a=7250 \mathrm{~km}, \exp \beta\left(r_{0}-a\right)=0.00876$, $x=\beta a e=2.2656>1, T_{S}=102 \mathrm{~min}$. So we use the following formula for $I_{n}(x)$ (Watson 1952; Li 2011b)

$$
\begin{aligned}
I_{n}(x)= & \frac{\exp x}{\sqrt{2 \pi x}}\left\{1-\frac{4 n^{2}-1}{1 !((8 x)}\right. \\
& \left.+\frac{\left(4 n^{2}-1\right)\left(4 n^{2}-3^{2}\right)}{1 ! 2 !(n+1)(n+2)}-\ldots\right\} .
\end{aligned}
$$

Substitution of $x$ into the above formula, yields: $I_{0}=$ 2.6990, $I_{1}=1.5584, I_{2}=0.9641, I_{3}=0.2827$. Substituting the above data into the above formulae (26), (27) and (28), we obtain the numerical results for the variable rate of semi-major axis and eccentricity. We can therefore compare the results for the electric induction drag with the atmosphere drag as shown in Table 2. It can be seen from Table 2 that the effect of electric induction drag is large compare with that of neutral atmosphere drag

(6) Comparison of the effect of the electric induction drag with that of Coulomb drag.

The formulae for the variable rate of the semi-major axis and eccentricity due to the action of Coulomb drag in the ionosphere was presented previously (Li 2011a):

$$
\begin{aligned}
& \frac{d a}{d t}=-2 C_{D i} \frac{\left(\pi R_{S}\right)^{2}}{m_{S} T_{S}} a^{2}\left(m_{i} N_{i}\right)\left(1+\frac{3}{4} e^{2}\right), \\
& \frac{d e}{d t}=-2 C_{D i} \frac{\left(\pi R_{S}\right)^{2}}{m_{i} T_{S}} a e\left(m_{i} N_{i}\right),
\end{aligned}
$$




$$
\frac{d T}{d t}=\frac{3}{2}\left(\frac{P}{a}\right) \frac{d a}{d t} .
$$

For the satellite at an altitude of 500-km, we use data based on Chapra (1961): $C_{D i}=0.32, N_{i}=2.51 \times 10^{7} \mathrm{~m}^{3}, m_{i}=$ $2.34 \times 10^{-26} \mathrm{~kg}, m_{i} N_{i}=5.85 \times 10^{-19} \mathrm{~kg} / \mathrm{m}^{3}, a=7250 \times$ $10^{3} \mathrm{~m}, e=0.025, m_{S}=45 \mathrm{~kg}, T_{S}=102 \mathrm{~min}, R_{S}=2 \mathrm{~m}$. Substituting these data into the above formulae (30)-(32), we obtain the numerical results for the semi-major axis and eccentricity. We also obtain comparison for the effects of the electric induction drag with Coulomb drag as shown in the Table 2 .

We can see from Table 2 that the effect of electric induction drag is smaller than that of atmospheric drag and larger than that of Coulomb drag on the rates of the semimajor axes and periods; The effect of electric induction drag is larger than that of Coulomb drag and smaller than that of the atmospheric drag for eccentricity rates in the same ionosphere.

\subsection{Conclusion}

We conclude that in the case of non- rotation of the Earth, the semi-major axis contracts gradually with time due to the electric induction drag, but does not have significant effect on eccentricity and inclination of the orbit as indicated in formula (15). In the case of rotation of the Earth, however, the semi-major axis and eccentricity increase gradually with time, but the increase is very small, and other orbital elements: argument or longitude of perigee, mean longitude at epoch, orbital inclination and ascending node exhibit no secular variation.
Open Access This article is distributed under the terms of the Creative Commons Attribution 4.0 International License (http://creative commons.org/licenses/by/4.0/), which permits unrestricted use, distribution, and reproduction in any medium, provided you give appropriate credit to the original author(s) and the source, provide a link to the Creative Commons license, and indicate if changes were made.

\section{References}

Allen, C.W.: Astrophysical Quantities, pp. 112-113. University of London, The Athlone Press, London (1973)

Chapra, K.P.: Interaction rapidly moving bodies on terrestrial atmosphere. Rev. Mod. Phys. 33, 152-198 (1961)

Lamb, H.: Hydrodynamics, Cambridge University Press, Cambridge (1932). Chap. X1, p. 589

Li, L.-S.: The lifetime of an artificial satellite moving in non-uniform rotating atmosphere and instantaneous circle orbit. Appl. Math. Mech. 12(5), 501-506 (1991)

Li, L.-S.: Perturbation effect of the Coulomb drag on the orbital elements of the Earth satellite in the ionosphere. Acta Astronaut. 68, 717-721 (2011a)

Li, L.-S.: Perturbation effects of quadratic drag on the orbital elements of a satellite in a central force field. J. Astronaut. Sci. 58(1), 23-33 (2011b)

Liu, L.: Orbital Mechanics for Artificial Earth Satellite, pp. 95-96. Beijing Higher Education Press, Beijing (1992)

Roy, A.E.: Orbital Motion, 3rd edn., p. 192. Adam Hilger, Bristol (1988). Chap. 6

Watson, G.N.: A Treatise on the Theory of Bessel Function, 2nd edn., p. 203. Cambridge University Press, Cambridge (1952)

Waytt, P.J.: Induction drag on a large negatively charged satellite moving in a magnetic-field-free ionosphere. J. Geophys. Res. 65, 1673-1678 (1960)

Zheng, X.-T., Ni, C.-X.: Celestial Mechanics and Dynamics of Astronomy, pp. 278-279. Beijing Normal University Press, Beijing (1989) 